\title{
Nitrite in saliva increases gastric mucosal blood flow and mucus thickness
}

Håkan Björne, Joel Petersson, Mia Phillipson, Eddie Weitzberg, Lena Holm, and Jon O. Lundberg

Original citation: J. Clin. Invest. 113:106-114 (2004). doi:10.1172/JCI200419019.

Citation for this erratum: J. Clin. Invest. 113:490 (2004). doi:10.1172/JCI200419019E1.

During the preparation of this manuscript for publication, errors were introduced into reference 58 . The correct reference appears below:

58. Forman, D., Al-Dabbagh, S., Doll, R. 1985. Nitrates, nitrites, and gastric cancer in Great Britain. Nature. 313:620-625.

\section{The adaptive imbalance in base excision-repair enzymes generates microsatellite instability in chronic inflammation}

Lorne J. Hofseth, Mohammed A. Khan, Mark Ambrose, Olga Nikolayeva, Meng Xu-Welliver, Maria Kartalou, S. Perwez Hussain, Richard B. Roth, Xiaoling Zhou, Leah E. Mechanic, Irit Zurer, Varda Rotter, Leona D. Samson, and Curtis C. Harris

Original citation: J. Clin. Invest. 112:1887-1894 (2003). doi:10.1172/JCI200319757.

Citation for this erratum: J. Clin. Invest. 113:490 (2004). doi:10.1172/JCI200419757E1.

In Figure 3, incorrect confidence intervals are shown. The correct figure appears below:

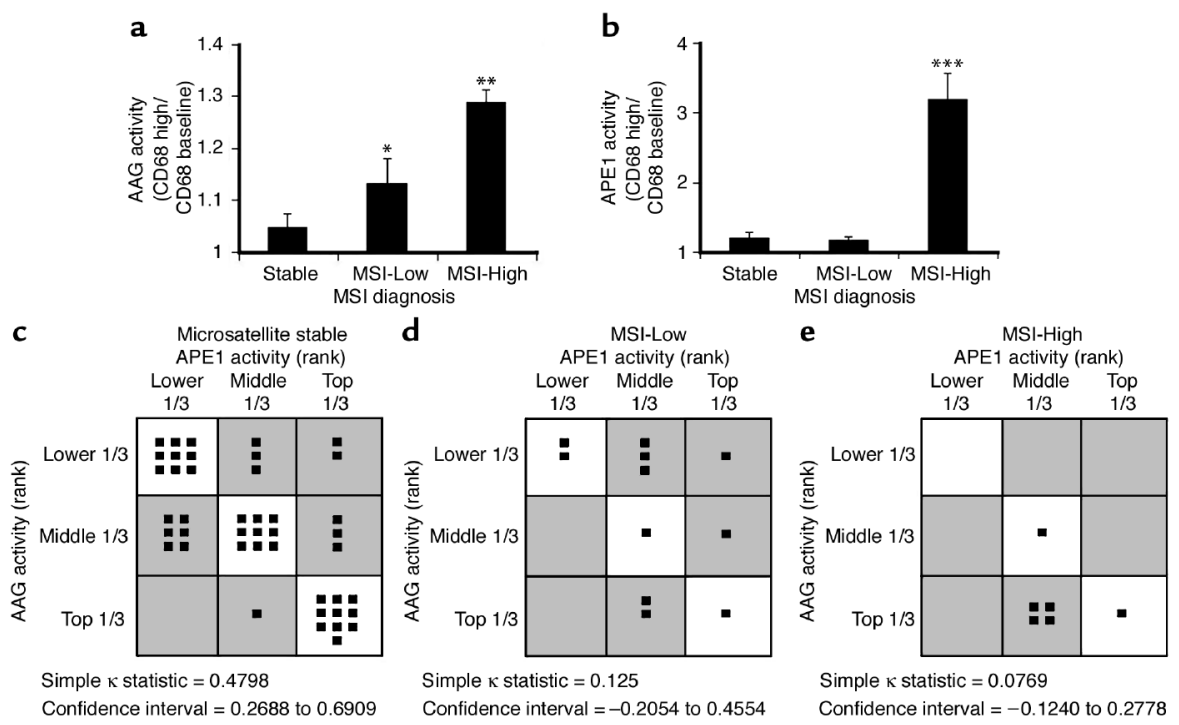

Figure 3

(a and $\mathbf{b}$ ) Correlation between MSI and AAG (a) or APE1 (b) activity. Bar graphs represent means \pm SEM. There was a significant trend for MSI and AAG activity (robust regression analysis, $P=0.0012$ ). Although this trend was not observed between MSI and APE1, there was a significant increase in APE1 activity in the MSI-High group $(n=5$; one-way ANOVA with Scheffe multiple comparison test, $P=0.0004)$. ${ }^{*}$, AAG activity is significantly higher in the MSI-Low group $(n=10)$ than in the microsatellite stable group $(n=15) .{ }^{*}$, AAG activity is significantly higher in the MSI-High group $(n=5)$ than in the MSI-Low group $(n=10) .{ }^{* *}$, APE1 activity is significantly higher in the MSI-High group $(n=5)$ than in the MSI-Low $(n=10)$ and microsatellite stable $(n=15)$ groups. (c-e) Number of samples belonging to a specific AAG and APE1 activity category. AAG and APE1 activities were ranked in order, then placed into tertiles as samples with activity belonging to the Lower $1 / 3$, Middle $1 / 3$, or Top $1 / 3$. (c) Of the 60 samples, 43 did not have a band shift and were characterized as microsatellite stable samples. (d) Of the 60 samples, 11 had a band shift in one of the markers examined (including TGF $\beta$ RII and BLM) and were characterized as MSI-Low samples. (e) Of the 60 samples, six had a band shift in two or more of the markers examined (including TGF $\beta$ RII and BLM) and were characterized as MSI-High samples. Shaded boxes represent activities where there is an imbalance of AAG and APE1 activities. The simple $\kappa$ statistic indicates a trend for imbalance between AAG and APE1 as MSI levels increase. The simple $\kappa$ statistic of 1.0 indicates no imbalance. A simple $\kappa$ statistic moving toward zero indicates greater imbalance between the two enzymes. 